\title{
Protocol to improve hypertension management in a VA outpatient clinic
}

\author{
Leo Gozdecki ${ }^{1,2}$, Holly Kramer (iD ${ }^{1,2,3 凶}$, Matthew Thomas ${ }^{1,2}$, Karam Gardezi ${ }^{1,2}$, Elizabeth Tarlov ${ }^{2}$, Ashley Hughes ${ }^{4}$, Kevin Stroupe ${ }^{1,2}$ and $^{\text {. }}$ \\ Meghan O'Halloran ${ }^{1,2}$
}

This is a U.S. government work and not under copyright protection in the U.S.; foreign copyright protection may apply 2022

This 20-week quality improvement study describes implementation of a hypertension identification and management program with use of a standardized oscillometric blood pressure (BP) measurement protocol, provider education, and audit/feedback of hypertension control in a Veterans Affairs primary care clinic. A total of 692 male Veterans ages 18-85 years with treated hypertension and at least one clinic visit in the previous year were included for analysis. Mean age was 69.7 years (standard deviation 7.6) and race and ethnicity were $42.0 \%$ White, $29.1 \%$ Black and $3.0 \%$ Hispanic. Prior to program implementation, clinic BP was measured using the auscultatory method with a manual syphgmomanometer. Baseline BP measurements demonstrated bias as determined by terminal digit preference for digits 0 and 8 in $29.5 \%$ and $25.2 \%$ of systolic (SBP) and $31.6 \%$ and $21.8 \%$ of diastolic BP measurements, respectively $(p<0.001)$. Post-implementation of the standardized oscillometric BP measurement protocol, digit preference was eliminated. Protocol compliance was $89.1 \%$ at 5 weeks and $92.4 \%$ at 20 weeks. Overall average SBP was significantly higher in the post-implementation period compared to average SBP in the 12-month pre-implementation period (137.4 [Standard Deviation (SD) 17.4] vs. 126.3 [SD 15.3]; $P<0.001$ ). Uncontrolled hypertension, (BP $\geq 140 / 90 \mathrm{mmHg}$ ), increased from $17.8 \%$ at baseline to $41.8 \%$ post-implementation while provider therapeutic inertia declined from $84.5 \%$ at baseline to $55.8 \%$ after 20 weeks. This study shows that terminal digit preference is reduced with implementation of standardized oscillatory BP measurement and a quality improvement program can reduce therapeutic inertia of hypertension treatment.

Journal of Human Hypertension (2023) 37:50-55; https://doi.org/10.1038/s41371-021-00650-0

\section{INTRODUCTION AND BACKGROUND}

Treating hypertension remains one of the most important interventions for preventing cardiovascular disease and mortality $[1,2]$. In order to effectively diagnose and treat hypertension, blood pressure (BP) must be measured with accuracy [3]. A recent scientific statement from the AHA/ACC recommends automated office blood pressure (AOBP) technique, which involves a fully automated BP measurement device that automatically obtains multiple BP measurements after a programmable rest period and displays the mean average values after all measurements are completed [3, 4]. Evidence for standardized BP procedure recommends that the average of at least two clinic BP measurements with digital sphygmomanometers separated by 1-min intervals reduces error compared to single manual BP measurements and should be used to assess hypertension and its control [3].

The hypertension improvement model endorsed by the AHA/ AMA, titled Measure Accurately, Act Rapidly and Partner with Patients (MAP), recommends AOBP for diagnosis and management of hypertension. MAP also provides education on hypertension treatment, interventions to reduce treatment inertia, and methods to partner with patients to improve medication compliance and lifestyle changes. The MAP program has demonstrated sustained improvements in BP control at 6 and 12 months when applied to underserved primary care clinics [5-7]. The initial MAP protocol studies conducted by Hanlin et al. and Egan et al., however, were completed prior to release of the 2017 ACC/AHA hypertension guideline and both studies targeted a goal BP of $<140 / 90 \mathrm{mmHg}$ for all patients [5, 7]. The ACC/AHA guideline recommends a BP goal $<130 / 80 \mathrm{mmHg}$ for persons with diabetes mellitus, chronic kidney disease, cardiovascular disease (CVD), 10year ASCVD risk $>10 \%$ and age $65-75$ years old. This ACC/AHA guideline recommendation was based on evidence that a lower BP goal in these populations reduces risk of stroke, coronary events, major cardiovascular events and cardiovascular mortality. For persons without these CVD risk criteria, the guideline recommends a BP goal <140/90 [2].

The objective of this quality improvement study was to pilot a hypertension intervention program modeled after the MAP program in a single Veterans Affairs Patient Aligned Care Team (PACT; i.e., VA's adaptation of the team-based Patient Centered Medical Home Model) [1]. We implemented an adapted MAP hypertension improvement program within a single VA PACT. This program educated clinic staff and providers to measure BP accurately and reliably using a standardized BP measurement protocol based on 2017 ACC/AHA guidelines. To reduce provider

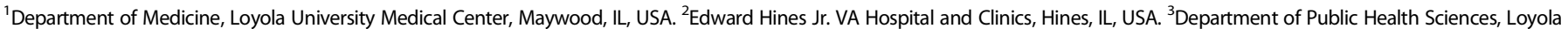
University Chicago, Maywood, IL, USA. ${ }^{4}$ Department of Biomedical and Health Information Sciences, School of Public Health, University of Illinois at Chicago, Chicago, IL, USA.

email: hkramer@lumc.edu

Received: 6 May 2021 Revised: 1 December 2021 Accepted: 15 December 2021

Published online: 24 January 2022 
inertia, providers were educated on guideline directed hypertension treatment and provided with patient-specific provider audit and feedback on performance of hypertension clinical management [1]. This study describes the quality improvement program and the impact of the interventions on BP control based on the ACC/AHA guideline and on therapeutic inertia. The primary goal was to improve hypertension control in an outpatient primary care setting.

\section{METHODS}

Design

This quality improvement initiative used monthly trend analysis from November 2019 to March of 2020 to track BP measurements, provider inertia and hypertension control. The study protocol was approved by the local institutional review board, which granted a waiver from documented informed consent.

\section{Setting}

This quality improvement project was implemented in a single PACT at the Edward Hines, Jr. VA Medical Center. This primary care PACT was exclusively staffed by 38 post-graduate year 1 through 3 Internal Medicine

\begin{tabular}{|c|c|}
\hline $\begin{array}{l}\mathrm{N}=2307 \\
\text { Veterans }\end{array}$ & $\begin{array}{l}\text {-2307 Veterans treated in clinic during July } 31,2018 \text { - } \\
\text { Augist } 1,2019\end{array}$ \\
\hline $\begin{array}{l}N=1181 \\
\text { Veterans } \\
\text { with htn }\end{array}$ & $\begin{array}{l}\text { - Excluded } 1126 \text { Veterans without hypertension diagnosis, } \\
\text { or with ugent clinic visit or end-stage renal disease or } \\
\text { enrolled in hospice }\end{array}$ \\
\hline $\begin{array}{l}\mathrm{N}=692 \\
\text { VA-MAP }\end{array}$ & - 489 Veterans without clinic visit during VA-MAP Program \\
\hline Participants & \\
\hline
\end{tabular}

Fig. 1 Flowchart of selection of Veterans included in analysis of VA-MAP. 2307 Veterans were treated in clinic during July 21, 2018August 1, 2019. We then excluded 1126 Veterans without a diagnosis of hypertension, urgent care visit, diagnosis of end-stage renal disease or receiving hospice care. Among the 1181 eligible Veterans, 692 had at least one clinic visit and a diagnosis of hypertension. resident physicians supervised by ten attending physicians. A group of eight resident physicians were supervised consistently by the same four attending physicians every 5 weeks. On average, 32 patients were treated in this clinic per day.

\section{Sample}

The study population was limited to Veterans between the age of 18 and 85 years old with an ICD10 diagnosis code of hypertension and at least one clinic visit in the previous academic year (July 31st 2018-August 1st 2019) with their assigned primary care resident physician. Patients with urgent care visits with providers other than the patient's primary care provider were not included in the study. Veterans were excluded if they were enrolled in hospice care or had a diagnosis of end-stage renal disease (ESRD) requiring dialysis. A total of 2307 patients were seen in the PACT during the preceding 12 months prior to start of VA-MAP and 1126 were excluded due to no previous diagnosis of HTN, urgent care visit, ESRD diagnosis or hospice status. Among the 1181 eligible Veterans, a total of 692 had at least one clinic visit within the VA-MAP study period and were included in the final analysis (Fig. 1).

\section{VA-MAP intervention}

The hypertension quality improvement protocol called VA-MAP was modeled off the original MAP design which has been previously described [6]. All members of the PACT team agreed to participate including registered nurses and licensed practical nurses (LPNs), pharmacists and physicians. Table 1 shows the components of the VA-MAP program and the PACT team members that implemented each component. Partnering with Patients, the final MAP component, included education on the importance of communicating with patients. The program was initiated on November 4, 2019 and was intended to last for at least 6 months but was terminated on March 9, 2020, due to the COVID19 pandemic and requisite clinic closure. Evidence-based tools and materials from the Target: BP website [1] were utilized to educate the PACT RN and LPN staff, all 38 resident providers, and the corresponding 10 attending physicians on the background and utility of the standardized BP measurement protocol including unattended (staff not in room when BP is measured) proper patient positioning and the importance of addressing therapeutic inertia and the 2017 ACC/AHA hypertension diagnosis/management guidelines with a discussion of the importance of patient engagement [2].

Prior to the start of VA-MAP, resident physicians were provided with baseline data on hypertension control and therapeutic inertia for their patient panel from the previous 12 -month academic year. All education was provided in a face-to-face format by the VA Chief Resident for Quality and Safety (CRQS) physician champion. One repeat education session in a face-to-face format was conducted at 10 weeks after the initial session. During these education sessions, resident physicians and their supervisors

Table 1. Components of the VA-MAP program.

\begin{tabular}{l} 
Target:BP AHA \\
Recommendations \\
Measure Accurately \\
- Use Proper Technique \\
- Take the measurements you \\
need to diagnose \\
- Prepare Your Patient \\
\hline Act Rapidly \\
- Reduce Therapeutic Inertia \\
- Collaborative Communication \\
Strategies
\end{tabular}

Partner with Patients

\section{Modified Implementation within VA Patient Aligned Care} Team (PACT)

- Training for LPNs on automated BP measurements and patient positioning (ask about empty bladder; cuff size check; bare arm; back and arm supported; arm at level of heart; legs uncrossed with feet flat on floor; refrain from talking)

- Repeat BP measurement triggered by BP reading $\geq 130 / 80$; BP measurements at least 1 min apart

- Posting AHA/AMA In-Office Measuring BP infographic

- All Resident Physician PCPs and Supervising Attendings undergo Face to Face orientation and didactic:

Review of In-Office BP Thresholds with resident physicians and supervising Attendings

- Baseline individualized reports reviewed with resident physicians

- Face to Face training on therapeutic inertia, including escalation of therapy strategies

- Audit \& Feedback with individualized resident physician PCP hypertension control rates report

- Implementation limited by COVID-19 pandemic

\section{Team Members}

- Chief Quality and Safety Resident with PACT Nurse Champion

- PACT LPN measures BP; documents and verbally communicates elevated readings to resident physician

- Chief Resident Quality \& Safety lead with support of:

- Resident Physician Champions (4)

- Associate Program Director

- Hand-off to Resident Physician Champion and Associate Program Director 
were also provided with individualized audit data. This feedback specifically included the percentage of patients at their target BP, as well as frequency of therapeutic inertia for those patients above their BP target. LPNs were responsible for obtaining patient vital signs and blood pressure in the clinic. Prior to the initiation of VA-MAP, LPNs measured a single seated BP manually and both systolic BP (SBP) and diastolic BP (DBP) were recorded with even numbers only. If the $B P$ was $\geq 140 / 90 \mathrm{mmHg}$ then a repeat manual BP was measured and recorded. For VA-MAP, LPNs were trained to adhere to a standardized BP measurement protocol with a screen and confirm approach [1]. The standardized BP protocol measurement involved placing the patient in a private room and seated with the back supported and feet flat on the floor. After a five-minute rest period, LPN staff were allowed to query patient's chief complaint, review their medications and complete other required clinical elements which may include screening for depression, alcohol use and pain. The LPN then initiated one digital BP measurement with a Dinamap V100 digital monitor. If the BP was $\geq 140 / 90 \mathrm{mmHg}$, then two additional blood pressure measured values with 1-min intervals while the patient was alone in the room. Both of these unattended BP measurements, as well as the initial measurement, were recorded and available for provider review. Providers were instructed to use the average of these three measurements when making clinical and treatment decisions. After 10 weeks of participation in VA-MAP, resident physicians were provided with updated data on hypertension control and therapeutic inertia, as well as re-education on hypertension management in a face-to-face meeting.

\section{Data collection and definitions}

Blood pressure control was defined as $<130 / 80 \mathrm{mmHg}$ for patients with any one of the following high CVD risk criteria listed in problem list or as a diagnostic billing code: age 65-75 years old, diabetes mellitus type 1 or 2, non-dialysis dependent chronic kidney disease stage 1-5, or CVD. CVD was defined as the diagnosis of either coronary artery disease, congestive heart failure, ischemic or hemorrhagic stroke, peripheral arterial disease, carotid artery disease or aortic aneurysm. For patients without any of these high risk criteria, BP control was defined as $<140 / 90 \mathrm{mmHg}$. Workflow compliance, therapeutic inertia and the percentage of patients with BP control was assessed every 5 weeks.

Workflow compliance with the VA-MAP BP measurement protocol was defined as the percentage of patients with an initial BP measurement $\geq 140 / 90 \mathrm{mmHg}$ who had a subsequent unattended BP measurement
VA-MAP were compared using a Chi-square test. Observational bias in BP measurement was assessed by examining terminal digit preference using a goodness of fit test with assumption of $10 \%$ frequency for digit 0 through 9 when using standardized BP measurement protocol. When assessing digit preference for manual BP measurements, an assumption of $20 \%$ frequency was used for digits $0,2,4,6$, and 8 . All statistical analyses were completed using STATA v 14.0. Statistical significance was set at $p<0.05$.

\section{RESULTS}

Table 2 provides the baseline characteristics of the 692 male Veterans included in the analyses. The mean age was 69.7 years (standard deviation [SD] 7.6) and $24.4 \%$ were 75 years and older. The majority of the patients (55.5\%) were age $65-75$ years old. All patients were male and race was $42.0 \%$ White, $29.1 \%$ Black, $6.7 \%$ Other race and $3.0 \%$ reported Hispanic ethnicity. Diabetes, cardiovascular disease and chronic kidney disease were present in $47.4 \%, 43.8 \%$ and $21.5 \%$ respectively. Overall, at least one high risk CVD criteria was present in 617 veterans (89.2\%).

Workflow compliance with the standardized BP measurement protocol during the quality improvement pilot is shown in Fig. 2. By week 5, compliance with the BP measurement protocol was $89.1 \%$ and this increased to $92.4 \%$ by week 20. As shown in Fig. 3, statistically significant digit preference was noted with both SBP and DBP measured manually prior to implementation of VA-MAP. Terminal digit preference for digits 0 and 8 , occurred in $29.5 \%$ and $25.2 \%$ of manual BP measurements for SBP and $31.6 \%$ and $21.8 \%$ of manual DBP measurements, respectively $(P<0.001$ for both SBP and DBP). Terminal digit preference for digits 4 and 6 were underrepresented for manual BP measurements for both SBP and DBP with frequencies between $10.1 \%$ and $11.9 \%$. No significant digit preference was noted with standardized digital BP measurement protocol.

Figure 4 shows that average SBP during the 20-week period of VA-MAP was significantly higher compared to average SBP during the 12 month period prior to initiation of VA-MAP (137.4 [Standard Deviation (SD) 17.4] vs. 126.3 [SD 15.3]; $P<0.001$ ). Similar findings

\# of patients with initial attended Screening BP $>140 / 90 \mathrm{mmHg}$ followed by unattended Confirmatory BP

\# of patients with initial attended screening $B P>140 / 90 \mathrm{mmHg}$

calculated as:

Patient demographics, chronic comorbid conditions, blood pressure, medication and dosage were extracted from the electronic medical record via manual chart review for both the baseline and post-intervention periods. Hypertension control rate was defined as total number of clinic patients with a diagnosis of hypertension in their profile and most recent clinic BP $<130 / 80 \mathrm{mmHg}$ for those with high-risk criteria and $<140 / 90$ $\mathrm{mmHg}$ for those without any high risk criteria divided by total number of patients with a diagnosis of hypertension in their profile.

Therapeutic inertia rate was defined as the total number of patient encounters with an BP measurement above goal without an escalation in BP lowering medication (increasing dose or adding a medication) divided by the total number of patient encounters with a BP measurement above goal. Therapeutic inertia rates for the pre-VA-MAP period were calculated using data from the most recent clinic visit prior to initiation of VA-MAP.

Data were collected utilizing a standardized form and all reviewers were trained with the form. Two reviewers reviewed the data points independently. Discrepancies were resolved by the physician lead for the project.

\section{Statistical analysis}

Characteristics of the Veterans who participated in the VA-MAP Program were examined with mean values and standard deviations for continuous variables and percentages for categorical variables. Blood pressure values pre- and post-implementation of VA-MAP were compared using a paired t-test. Prevalence of hypertension control and provider inertia during the 12-month period before and during the 20-week period after initiation of were noted with DBP post vs. pre VA-MAP (76.6 [SD 10.3] vs. 74.0 [SD 9.9]; $P<0.001)$.

At baseline, $17.8 \%$ of Veterans overall had a blood pressure $\geq 140 / 90 \mathrm{mmHg}$; BP was $\geq 130 / 80 \mathrm{mmHg}$ in $49.8 \%$ of the Veterans with at least one high risk CVD criteria and $\geq 140 / 90 \mathrm{mmHg}$ in $24.0 \%$ among Veterans without any high-risk CVD criteria. After initiation of VA-MAP, $41.8 \%$ of the Veterans overall had an average $\mathrm{BP} \geq 140 / 90 \mathrm{mmHg} ; 62.6 \%$ of Veterans with at least one high risk CVD criteria had a BP $\geq 130 / 80 \mathrm{mmHg}$ and $42.7 \%$ of Veterans without high risk CVD criteria had a $B P \geq 140 / 90 \mathrm{mmHg}$. Therapeutic inertia declined from $84.5 \%$ at baseline to $55.8 \%$ after 20 weeks of VA-MAP implementation. (Fig. 5).

\section{DISCUSSION}

This pilot program modeled after MAP from the Target: BP program [1], utilized a multi-faceted approach of education, training, provider audit and feedback, and standardized BP measurement protocol to improve hypertension treatment. This pilot study shows that a multi-faceted quality improvement program for hypertension can be successfully implemented in a VA PACT and sustained for at least 20 weeks. Consistent with previous studies, use of a standardized BP measurement protocol led to decreased digit preference, a marker of observer bias $[8,9]$. In contrast, higher average BP measurements were observed in 
Table 2. Baseline characteristics of the Veterans in VA-MAP.

\begin{tabular}{|c|c|}
\hline & $\begin{array}{l}\text { Veterans with hypertension } \\
N=692\end{array}$ \\
\hline${ }^{*}$ Mean age, years & $69.7(7.6)$ \\
\hline \multicolumn{2}{|l|}{ Age Group } \\
\hline Age <65 years, $N(\%)$ & $139(20.1)$ \\
\hline Age $65-74$ years, $N(\%)$ & $384(55.5)$ \\
\hline Age $75+$ years, $N(\%)$ & $169(24.4)$ \\
\hline Male, $N(\%)$ & $692(100)$ \\
\hline \multicolumn{2}{|l|}{ Race/ethnicity } \\
\hline White, $N(\%)$ & $444(64.2)$ \\
\hline Black, $N(\%)$ & $201(29.1)$ \\
\hline Other, $N(\%)$ & $47(6.7)$ \\
\hline \multicolumn{2}{|l|}{ Ethnicity } \\
\hline Hispanic, $N(\%)$ & $21(3.0)$ \\
\hline \multicolumn{2}{|l|}{ Diagnosed chronic conditions } \\
\hline Diabetes mellitus, $N(\%)$ & $328(47.4)$ \\
\hline Chronic kidney disease, $N$ (\%) & $149(21.5)$ \\
\hline Cardiovascular disease, $N(\%)$ & $303(43.8)$ \\
\hline+ High risk CVD criteria, $N(\%)$ & $617(89.2)$ \\
\hline *Systolic blood pressure $\mathrm{mmHg}$ & $126.3(15.3)$ \\
\hline${ }^{*}$ Diastolic blood pressure $\mathrm{mmHg}$ & $74.0(9.9)$ \\
\hline $\mathrm{BP}<140 /<90 \mathrm{mmHg}, N(\%)$ & $569,82.2 \%$ \\
\hline $\begin{array}{l}\mathrm{BP} \geq 140-159 / \geq 90-99 \mathrm{mmHg} \\
N(\%)\end{array}$ & $79(11.4)$ \\
\hline $\mathrm{BP} \geq 160 / \geq 100 \mathrm{mmHg}, N(\%)$ & $44(6.4)$ \\
\hline \multicolumn{2}{|c|}{$\begin{array}{l}\text { *Shown as mean (standard deviation); +High risk CVD (cardiovascular } \\
\text { disease) criteria defined as presence of diabetes, chronic kidney disease } \\
\text { cardiovascular disease, or age } 65-75 \text { years: } \mathrm{BP}=\text { blood pressure }\end{array}$} \\
\hline \multicolumn{2}{|l|}{$\begin{array}{r}100 \% \\
90 \%\end{array}$} \\
\hline \multicolumn{2}{|l|}{$\mathbf{8 0 \%}$} \\
\hline \multicolumn{2}{|l|}{$70 \%$} \\
\hline \multicolumn{2}{|l|}{$60 \%$} \\
\hline \multicolumn{2}{|l|}{$50 \%$} \\
\hline \multicolumn{2}{|l|}{$40 \%$} \\
\hline \multicolumn{2}{|l|}{$30 \%$} \\
\hline \multicolumn{2}{|l|}{$20 \%$} \\
\hline \multicolumn{2}{|l|}{$10 \%$} \\
\hline 10 wks & 15 wks \\
\hline
\end{tabular}

Fig. 2 Compliance with screen/confirm protocol for BP measurement by time after implementation. The compliance increased from $89.1 \%$ after 5 weeks to $92.4 \%$ after 20 weeks.

the clinic population after VA-MAP. Most studies have shown reductions in average SBP with use of AOBP compared to manual $B P$ measurements $[5,10,11]$, and this reduction has been largely attributed to mitigation of white coat effect. Our finding of higher BP with standardized BP measurement protocol vs. manual BP measurement has been reported by one other published study which found lower estimates of BP control in four out of ten clinics after implementation of a quality improvement program for hypertension which included AOBP [12]. Lower rates of BP control in these four clinics were attributed to attenuation of digit preference in that study $[12,13]$. The increase in BP post implementation of the VA-MAP program could be attributed to
Digit preference with manual systolic and diastolic blood pressure measurements

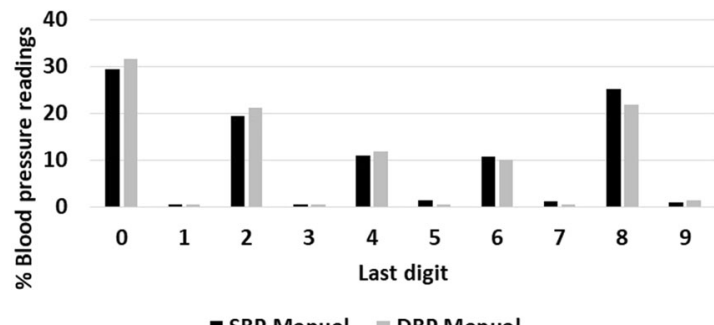

- SBP Manual DBP Manual

Digit preference with Dinamap V100 digital monitor systolic and diastolic blood pressure measurements

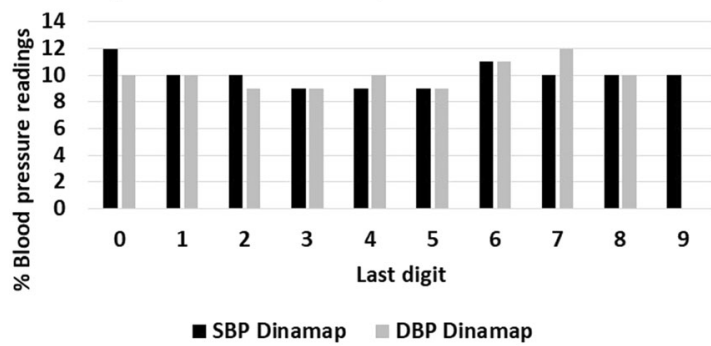

Fig. 3 Digit preference with manual systolic and diastolic blood pressure measurements. Top figure shows digit preference with manual blood pressure measurements. Bottom figure shows digit preference with blood pressure measurement using a Dinamap V100 digital monitor.

reduction of terminal digit preference in BP measurement. However other factors such as changes in medication compliance after implementation of the VA-MAP protocol could be operative.

The VA/Department of Defense Clinical Practice Guideline for the Diagnosis and Management of Hypertension in the Primary Care Setting states that AOBP with an average of three BP measurements is the preferred method for BP measurement while manual techniques with an average of 2 or more readings may also be utilized in outpatient settings [14]. Currently, outpatient clinics in the U.S. Veterans Administration Medical Centers as well as other non-VA medical centers and clinics continue to manually measure $\mathrm{BP}$ or confirm BP readings from automated devices with manual BP measurements. Our study shows that lack of standardized protocol with the use of manual BP measurement may introduce bias in estimation of BP. Barriers to implementation of a standardized digital BP measurement protocol into clinical practice include lack of equipment and training on digital devices and protocols along with perceived time constraints related to obtaining multiple BP measurements. While we did not measure LPN workflow time, we found that the standardized BP measurement and protocol could be implemented and sustained in a busy outpatient clinic as demonstrated by the sustained workflow protocol fidelity at 20 weeks. Use of a screen and confirm approach helped to reduce work burden for clinic nurses. Physician inertia for hypertension treatment decreased and this is likely due to monitoring and reporting of physician treatment inertia. Future studies will need to examine whether a hypertension improvement program can be sustained within VA clinics for a longer period of time and whether interventions to partner with patients can be successfully included to increase medication compliance.

This study was limited by the fact that the VA-MAP program was piloted in a single clinic and was terminated at 20 weeks which precluded determination of program sustainment over a longer time frame. Dinamap V100 digital BP measurement devices were used for BP measurement which do not have programmable rest periods and do not calculate an average of multiple BP readings. 

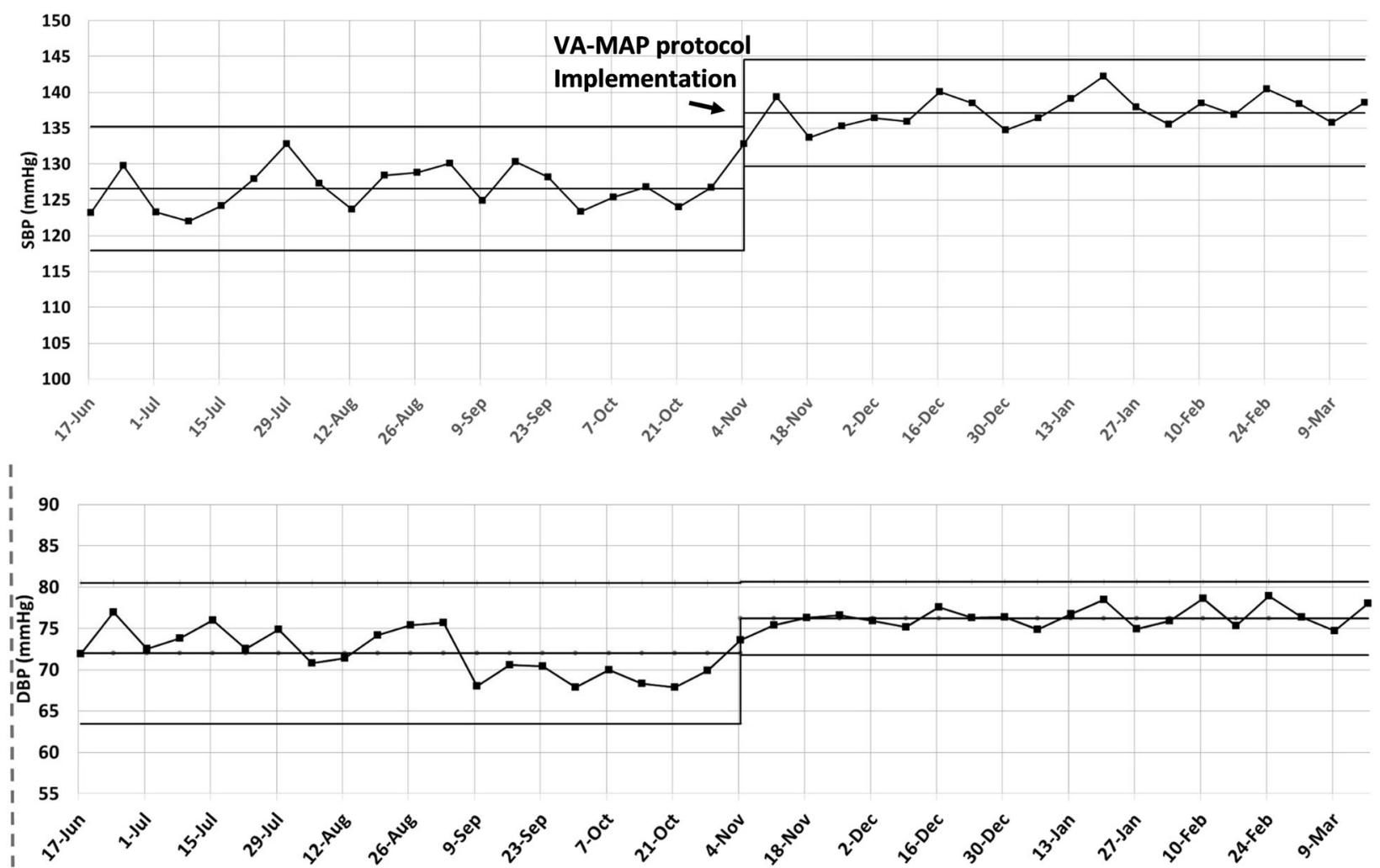

Fig. 4 Average clinic systolic and diastolic blood pressure in the 20 weeks before and after implementation of the VA-MAP intervention. Top figure shows average systolic blood pressure among clinic patients before and after implementation of the VA-MAP intervention. Bottom figure shows average diastolic blood pressure in clinic patients before and after implementation of the VA-MAP intervention.

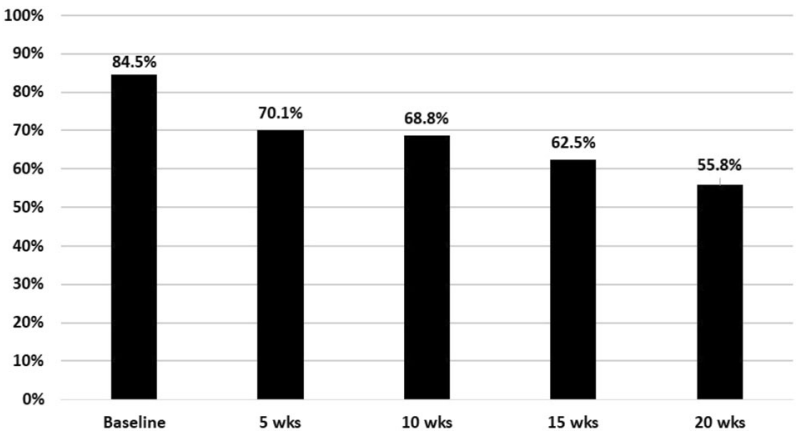

Fig. 5 Provider inertia in the 20 weeks before and after implementation of the VA-MAP intervention. The provider inertia for escalating blood pressure lowering medications for uncontrolled hypertension was $84.5 \%$ at baseline. After 20 weeks of the VA-MAP intervention, provider inertia declined to $55.8 \%$.

In addition, the program did not reflect a complete MAP program because it did not include partnering with patient interventions beyond provider education, therefore coordinated interventions with nurse educators, pharmacists, dietitians, and home telehealth are essential next steps. Future interventions for BP control should consider these PACT resources to improve medication compliance and patient and caregiver partnership.

In summary, this quality improvement pilot study based on MAP shows that a standardized BP measurement protocol can be implemented and sustained for at least 20 weeks in a VA PACT setting. Use of a standardized BP measurement protocol reduces terminal digit preference which may result in a more accurate representation of hypertension prevalence. The use of audit and feedback techniques to educate and train physicians on BP control can reduce therapeutic inertia. These data may help other VA PACTs or non-VA clinics develop quality improvement programs aimed to improve the detection and treatment of hypertension.

\section{Summary table}

What is known on this topic

- The hypertension improvement model titled Measure Accurately, Act Rapidly and Partner with Patients (MAP) recommends use of electronic blood pressure measurement for diagnosis and management of hypertension and provides education and interventions to reduce treatment inertia.

- The MAP program has been shown to improve blood pressure control when implemented in underserved primary care clinics.

- It remains unclear whether an intervention modeled after the MAP Program can improve hypertension control in a Veterans Affairs outpatient patient aligned clinical care team (PACT).

What this study adds

- A pilot hypertension improvement program modeled after MAP can be successfully implemented in a Veterans Affairs PACT and reduce hypertension treatment inertia.

- Use of a screen and confirm approach helps to reduce work burden for clinic nurses and sustain a standardized blood pressure measurement protocol using electronic devices in a busy outpatient clinic.

- Digit bias with use of manual blood pressure measurements can be eliminated by measuring blood pressure with electronic devices.43 


\section{REFERENCES}

1. AnonymousTARGET:BP. https://targetbp.org/blood-pressure-improvement-program/ control-bp/. Accessed 1 Mar 2021.

2. Whelton PK, Carey RM, Aronow WS, Casey DE Jr, Collins KJ, Dennison Himmelfarb C, et al. 2017 ACC/AHA/AAPA/ABC/ACPM/AGS/APhA/ASH/ASPC/NMA/PCNA Guideline for the prevention, detection, evaluation, and management of high blood pressure in adults: a report of the American College of Cardiology/American Heart Association Task Force on Clinical Practice Guidelines. J Am Coll Cardiol. 2018;71:e127-e248.

3. Muntner P, Shimbo D, Carey RM, Charleston JB, Gaillard T, Misra S, et al. Measurement of blood pressure in humans: a scientific statement from the American Heart Association. Hypertension. 2019;73:e35-e66.

4. Roerecke M, Kaczorowski J, Myers MG. Comparing automated office blood pressure readings with other methods of blood pressure measurement for identifying patients with possible hypertension: a systematic review and metaanalysis. JAMA Intern Med. 2019;179:351-62.

5. Egan BM, Sutherland SE, Rakotz M, Yang J, Hanlin RB, Davis RA, et al. Improving hypertension control in primary care with the measure accurately, act rapidly, and partner with patients protocol. Hypertension. 2018;72:1320-7.

6. Boonyasai RT, Rakotz MK, Lubomski LH, Daniel DM, Marsteller JA, Taylor KS, et al. Measure accurately, act rapidly, and partner with patients: an intuitive and practical three-part framework to guide efforts to improve hypertension control. J Clin Hypertens. 2017;19:684-94.

7. Hanlin RB, Asif IM, Wozniak G, Sutherland SE, Shah B, Yang J, et al. Measure accurately, act rapidly, and partner with patients (MAP) improves hypertension control in medically underserved patients: Care Coordination Institute and American Medical Association Hypertension Control Project Pilot Study results. J Clin Hypertens. 2018;20:79-87.

8. Ataman SL, Cooper R, Rotimi C, McGee D, Osotimehin B, Kadiri S, et al. Standardization of blood pressure measurement in an international comparative study. J Clin Epidemiol. 1996;49:869-77.

9. Thavarajah S, White WB, Mansoor GA. Terminal digit bias in a specialty hypertension faculty practice. J Hum Hypertens. 2003;17:819-22.

10. Walsh JM, McDonald KM, Shojania KG, Sundaram V, Nayak S, Lewis R, et al. Quality improvement strategies for hypertension management: a systematic review. Med Care. 2006;44:646-57.

11. Myers MG, Godwin M, Dawes M, Kiss A, Tobe SW, Grant FC, et al. Conventional versus automated measurement of blood pressure in primary care patients with systolic hypertension: randomised parallel design controlled trial. BMJ. 2011;342: d286
12. Khan T, Yang J, Barkowski L, Tapper B, Lubomski L, Daniel D, et al. A hypertension control quality improvement pilot program: Experiences and blood pressure outcomes from physician practices. Int J Healthc. 2018;4:42-49.

13. Turner MJ, Speechly C, Bignell N. Sphygmomanometer calibration-why, how and how often? Aust Fam Physician. 2007;36:834-8.

14. Anonymous. VA/DoD Clinical Practice Guideline. Diagnosis and Management of Hypertension in the Primary Care Setting. The Diagnosis and Management of Hypertension in the Primary Care Setting Work Group With support from: The Office of Quality, Safety and Value, VA, Washington, DC \& Office of Evidence Based Practice, U.S. Army Medical Command. March, 2020.

\section{AUTHOR CONTRIBUTIONS}

LG-conceptual design, data collection, supervision, manuscript writing and editing HK-conceptual design, data analysis, manuscript writing and editing; MT-data collection, manuscript writing and editing; KG—-data collection, manuscript writing and editing; $\mathrm{ET}-$ manuscript writing and editing; $\mathrm{AH}-$ manuscript writing and editing; KS-manuscript writing and editing; $\mathrm{MO}$-conceptual design, data collection, supervision, manuscript writing and editing.

\section{FUNDING}

Small Award Initiative For Impact by the Center of Innovation for Complex Chronic Healthcare (CINCCH), Hines VA (PIs MO and ET).

\section{COMPETING INTERESTS}

Dr. Kramer is a consultant for Bayer pharmaceuticals. The other authors have no financial disclosures of conflict of interest

\section{ADDITIONAL INFORMATION}

Correspondence and requests for materials should be addressed to Holly Kramer.

Reprints and permission information is available at http://www.nature.com/ reprints

Publisher's note Springer Nature remains neutral with regard to jurisdictional claims in published maps and institutional affiliations. 